\title{
Non Vitamin K Antagonist Oral Anticoagulants in Atrial Fibrillation Patients Scheduled for Electrical Cardioversion: A Real-Life Propensity Score Matched Study
}

\author{
Anna Rago' \\ Enrica Pezzullo' \\ Marco Malvezzi Caracciolo d'Aquino' \\ Gabriella Scognamiglio' \\ Valentina Maria Caso (iD) ${ }^{2}$ \\ Francesco Martone ${ }^{3}$ \\ Emilio Attena ${ }^{4}$ \\ Valentina Parisi ${ }^{5}$ \\ Antonio D'Onofrio ${ }^{4}$ \\ Paolo Golino' \\ Gerardo Nigro (iD) \\ Vincenzo Russo' \\ 'Department of Cardiology, Monaldi \\ Hospital, Naples, Italy; ${ }^{2}$ Department of \\ Medical Translational Sciences, University \\ of the Study of Campania "Luigi \\ Vanvitelli”, Naples, Italy; ${ }^{3}$ Clinical \\ Biochemistry Unit, Monaldi Hospital, \\ Naples, Italy; ${ }^{4}$ Departmental Unit of \\ Electrophysiology, Monaldi Hospital, \\ Naples, Italy; ${ }^{5}$ Department of \\ Translational Medical Sciences, University \\ of Naples Federico II, Naples, Italy
}

Aim: The aim of the present study was to assess the safety and effectiveness of non-vitamin $\mathrm{K}$ antagonist oral anticoagulants (NOACs) versus vitamin $\mathrm{K}$ antagonists (VKAs) in atrial fibrillation (AF) patients undergoing electrical cardioversion (EC).

Methods: A propensity score-matched analysis was performed in order to identify two homogeneous groups including AF patients on NOACs and VKAs treatment scheduled for EC. The primary safety endpoint was major bleeding. The composite of stroke, transient ischemic attack (TIA) and systemic embolism (SE) was the primary effectiveness endpoint. The discontinuation rate of anticoagulant therapy was assessed.

Results: A total of $495 \mathrm{AF}$ patients on NOACs therapy and scheduled for $\mathrm{EC}$ were compared to 495 VKAs recipients. No statistically significant differences in the incidence of both major bleeding $(1.01 \%$ versus $1.4 \% ; P=0.5)$ and thromboembolic events $(0.6 \%$ versus $0.8 \% ; P=0.7$ ) were observed during a mean follow-up of $15 \pm 3$ months. The discontinuation rate of NOACs was significantly lower compared to VKAs $(1.6 \%$ versus $3.6 \%, P=0.04)$.

Conclusion: We showed a safe and effective clinical profile of NOACs among AF patients scheduled for electrical cardioversion in real-life setting. Patients on NOACs therapy showed a lower discontinuation rate compared to those on VKAs.

Keywords: atrial fibrillation, electrical cardioversion, transesophageal echocardiogram, nonvitamin $\mathrm{K}$ antagonist oral anticoagulants, discontinuation rate, vitamin $\mathrm{K}$ antagonists

\section{Introduction}

An increased risk of peri-procedural thromboembolic events was shown among atrial fibrillation (AF) patients undergoing electrical cardioversion; ${ }^{1}$ in particular it is about $7 \%$ in those who underwent cardioversion without adequate anticoagulation. The safety and efficacy of non-vitamin $\mathrm{K}$ antagonist oral anticoagulants (NOACs) in AF patients have been demonstrated in large randomized clinical trials $(\mathrm{RCTs})^{2-5}$ and real-world observational studies, ${ }^{6-10}$ resulting in a rapid increase of their use across different clinical scenarios. ${ }^{11-19}$ Both RCTs post-hoc analyses ${ }^{20-23}$ and observational studies ${ }^{24-26}$ evaluating the periprocedural use of NOACs in AF patients undergoing EC showed a similar risk of thromboembolic and bleeding events compared to vitamin $\mathrm{K}$ antagonists (VKAs). The current guidelines recommend the early use of NOACs before every AF cardioversion. For patients with AF
Correspondence: Vincenzo Russo Sciences, University of Campania -

Monaldi Hospital, Via Leonardo Bianchi,

Naples, 80131, Italy

Fax +39-0817587482

Email v.p.russo@libero.it 
lasting more than 48 hours the oral anticoagulation is recommended at least 3 weeks before cardioversion and for at least 4 weeks afterwards. ${ }^{27,28}$ Actually, little is still known about the clinical outcomes of AF patients on NOACs therapy undergoing EC. ${ }^{29-33}$ The aim of our study was to assess the safety and effectiveness of NOACs compared to VKAs treatment in AF patients scheduled for transesophageal echocardiography (TEE)guided EC.

\section{Materials and Methods}

All consecutive AF patients followed at our Institution were prospectively included in the multicenter Atrial Fibrillation Research Database, which actually includes 7230 AF patients. Among this population, we retrospectively analyzed a cohort of 1510 patients (ambulatory setting) scheduled for TEE-guided EC at our InstitutionUniversity of Campania "Luigi Vanvitelli", Naples from September 2017 to September 2019. All AF patients included in the Database were on oral anticoagulant treatment, both NOACs and VKAs. The indication to NOACs or VKAs was based on treating physician's decision. All AF patients were OAC treatment naïve at inclusion and were treated on oral anticoagulants at least 3 weeks before cardioversion.

At each 6 months follow-up visit, during a mean follow up of was $15 \pm 3$ months, the clinical status, occurrence of stroke, transient ischemic attack (TIA), systemic embolism (SE), myocardial infarction, major bleeding (MB), clinically relevant non-major bleedings (CRNMB) or other side effects were assessed. Patients with a diagnosis of persistent non-valvular AF (duration $>48 \mathrm{~h}$ ), aged $\geq 18$ years and without contraindications to oral anticoagulant therapy were eligible for inclusion in the study. Patients with a follow-up less than one year (n:11), patients on VKAs treatment with time in therapeutic range (TTR) lower than 70\% (n:29) and patients with previous left atrial thrombosis or gastrointestinal major bleeding history were excluded from the study.

The electrical cardioversions were electively performed in electrophysiology operating room through biphasic DC shock from 150 to 200 Joules, depending on the patients weight, in deep sedation with propofol administered with the anesthetist support. Adherence to treatment was defined by using the cutoff point for proportion of days covered $\geq 80 \%$ from the first anticoagulants prescription; in presence of proportion of days on anticoagulant treatment $\leq 80 \%$ we considered a therapy discontinuation. The adherence to treatments was checked before and after cardioversion through an accurate anamnesis. The most frequent causes of anticoagulant treatment discontinuation were dyspepsia, gastrointestinal disorders and poor compliance.

Two groups (NOACs versus VKAs) without significant differences in baseline characteristics were generated by propensity score matching analysis (Table 1). The occurrence of major bleedings (MBs), defined according to the International Society of Hemostasis and Thrombosis (ISTH) criteria, ${ }^{34}$ and of thromboembolic events, defined as composite of ischemic stroke, systemic embolism and transient ischemic attack, were considered the primary safety and effectiveness endpoints, respectively. Ischemic stroke was defined as an event of neurologic deficit in the absence of an intracranial hemorrhage, diagnosed by cerebral computerized tomography and lasting more than 24 hours. TIA was considered as a temporary neurologic deficit lasting more than 24 hours. SE was defined as an extremity or organ acute vascular occlusion. ${ }^{34}$ The secondary safety and effectiveness endpoints included all-cause mortality and the clinically relevant non-major bleeding (CRNMB) events, respectively. CRNMB was defined as a clinically evident bleeding not meeting the criteria for MB but requiring medical intervention, pain, unscheduled contact (visit or telephone) with a physician, temporary interruption of study drug (ie, delayed or missed dosing) or impairment of daily activities according to ISTH criteria. ${ }^{34}$ The institutional review committee of University of Campania - Monaldi Hospital approved the database and the present analysis.

\section{Statistical Analysis}

Categorical data were expressed as number and percentage, while continuous variables either as median and interquartile range [IQR] or mean and standard deviation (SD), based on their distribution assessed both by the Kolmogorov-Smirnov and the Shapiro-Wilk tests. Between group differences, for categorical variables, were assessed by the Chi-square test, as the sample size was $>50$ subjects, with the application of Yates correction where appropriate. Either parametric Student $t$ test or nonparametric Mann-Whitney $U$-Test and Wilcoxon test were instead used to compare continuous variables, according to their distribution. The nearest neighbor propensity score matching with 1:1 ratio was used to minimize bias between NOACs and VKAs groups. The variables included in the propensity score were: age, female sex, 
Table I Baseline Clinical Characteristics of the Study Population Before and After Propensity Score Matching

\begin{tabular}{|c|c|c|c|c|c|c|}
\hline \multirow[t]{2}{*}{ Variable } & \multicolumn{3}{|c|}{ Before Propensity Score Matching } & \multicolumn{3}{|c|}{ After Propensity Score Matching } \\
\hline & NOACs $(n=825)$ & VKAs $(n=625)$ & P-value & NOACs $(n=495)$ & VKAs $(n=495)$ & P-value \\
\hline Age (years) & $64.1 \pm 10.4$ & $73.9 \pm 6.4$ & $<0.001$ & $63.3 \pm 10.1$ & $64.1 \pm 9.9$ & 0.73 \\
\hline Female (\%) & 45.4 & 43.1 & 0.58 & 43.5 & 42.8 & 0.72 \\
\hline BMI $\left(\mathrm{kg} / \mathrm{m}^{2}\right)$, & $26.8 \pm 5.9$ & $28.8 \pm 7.1$ & 0.82 & $25.9 \pm 8.3$ & $26.4 \pm 7.1$ & 0.77 \\
\hline Hypertension (\%) & 48.6 & 58.2 & 0.001 & 50.2 & 53.1 & 0.57 \\
\hline CHA2DS2VASc score & $2.6 \pm 1.4$ & $3.2 \pm 1.5$ & 0.02 & $2.3 \pm 0.4$ & $2.2 \pm 0.5$ & 0.56 \\
\hline HAS-BLED score & $2.5 \pm 1.1$ & $3.3 \pm 1.0$ & 0.001 & $2.4 \pm 1.1$ & $2.1 \pm 1.5$ & 0.55 \\
\hline Diabetes Mellitus (\%) & 16 & 25 & 0.06 & 13 & 14 & 0.4 \\
\hline Heart failure (\%) & 17.8 & 27.2 & 0.002 & 22.5 & 23.3 & 0.8 \\
\hline Previous stroke/TIA (\%) & 27.8 & 37.4 & 0.001 & 28.8 & 29.3 & 0.6 \\
\hline Previous MI (\%) & 8.2 & 13.1 & 0.01 & 5.6 & 6.2 & 0.7 \\
\hline $\mathrm{CrCl}$ (milliliter/minute) & $71.3 \pm 12.1$ & $60.5 \pm 12.9$ & 0.001 & $70.3 \pm 21.1$ & $71.2 \pm 21.2$ & 0.7 \\
\hline Left atrial diameter (millimeters) & $45.3 \pm 5.7$ & $46.7 \pm 5.6$ & 0.8 & $46.2 \pm 4.3$ & $47.1 \pm 5.4$ & 0.9 \\
\hline LAVI (milliliter/meter $\left.{ }^{2}\right)$ & $32.2 \pm 1.1$ & $33.4 \pm 1.5$ & 0.42 & $32.4 \pm 2.4$ & $33.1 \pm 1.2$ & 0.7 \\
\hline LV EF (\%) & $53.2 \pm 6.4$ & $44.3 \pm 6.1$ & 0.002 & $55.3 \pm 5.1$ & $54.4 \pm 3.8$ & 0.8 \\
\hline TEE performed (\%) & 100 & 100 & & 100 & 100 & \\
\hline Antiplatelets (\%) & 21 & 18 & 0.8 & 20 & 19 & 0.8 \\
\hline PPI (\%) & 88 & 85 & 0.5 & 86 & 84 & 0.5 \\
\hline Beta-blockers (\%) & 90 & 92 & 0.5 & 89 & 87 & 0.5 \\
\hline ACE-I or ARBs (\%) & 75 & 77 & 0.5 & 74 & 73 & 0.5 \\
\hline
\end{tabular}

Notes: Values are expressed as mean \pm SD unless otherwise stated.

Abbreviations: NOACs, non-vitamin K antagonist oral anticoagulants; SD, standard deviation; BMI, body mass index; LAVI, Indexed left atrial volume; MI, myocardial infarction; LV EF, left ventricular ejection fraction; TEE, transesophageal echocardiogram; TIA, transient ischemic attack; CrCl, creatinine clearance; PPI, proton pump inhibitors; ACE-I, angiotensin converting enzyme inhibitors; ARBs, angiotensin II receptors blockers.

BMI, hypertension, CHA2DS2-VASc score, HAS-BLED score, diabetes mellitus, heart failure, prior stroke/TIA, prior myocardial infarction (MI), glomerular filtration rate, left atrial diameter, indexed left atrial volume and left ventricular ejection fraction. All collected covariates were defined at inclusion according to clinical criteria. Persisting significant differences in baseline characteristics after matching were checked. For all test, a p value $<0.05$ was considered statistically significant. All statistical analyses were performed using RStudio (RStudio Team (2016). RStudio: Integrated Development for R. RStudio, Inc, Boston, MA URL http://www.rstudio.com/.)

\section{Results}

1510 consecutive AF patients scheduled for TEE-guided EC and who received NOACs (n: 825) or VKAs therapy (n: 625) have been analyzed. 495 NOACs [24\% in dabigatran (DAB),26\% in apixaban (API), 28\% in edoxaban (EDO), 22\% in rivaroxaban (RIVA) treatment]and 495 VKAs recipients with similar demographic and clinical characteristics were identified by propensity score matching. Table 1 shows the baseline characteristics of study population before and after propensity score matching. The mean follow-up was $15 \pm 3$ months for both NOACs and VKA groups. 11 patients with a follow up of $<1$ year were excluded to perform an accurate analysis of the longterm safety and effectiveness outcomes of AF patients on anticoagulant therapy. None of them experienced thromboembolic or bleeding events. 29 VKAs patients with time in therapeutic range lower than $70 \%$ were excluded in order to eliminate the possible bias due to a not well controlled VKAs treatment. After PSM, all AF patients were taking only full dosages of NOACs.

A left atrial appendage thrombus was revealed by TEE in four patients $(0.4 \%)$ in whom the EC was not performed and they were excluded from the analysis. After PSM, all AF patients were taking only full dosages of NOACs. Time in therapeutic range did not vary between VKA users and did not affect results.

These patients presented high thromboembolic risk and moderate renal impairment: two patients $(0.4 \%)$ in the NOACs group $\left[\mathrm{CHA}_{2} \mathrm{DS}_{2} \mathrm{VASc}\right.$ score: 5 and creatinine clearance ( $\mathrm{CC}$; calculated by Cockcroft-Gault Equation): 36 milliliter/minute in one patient in RIVA therapy; $\mathrm{CHA}_{2}$ 


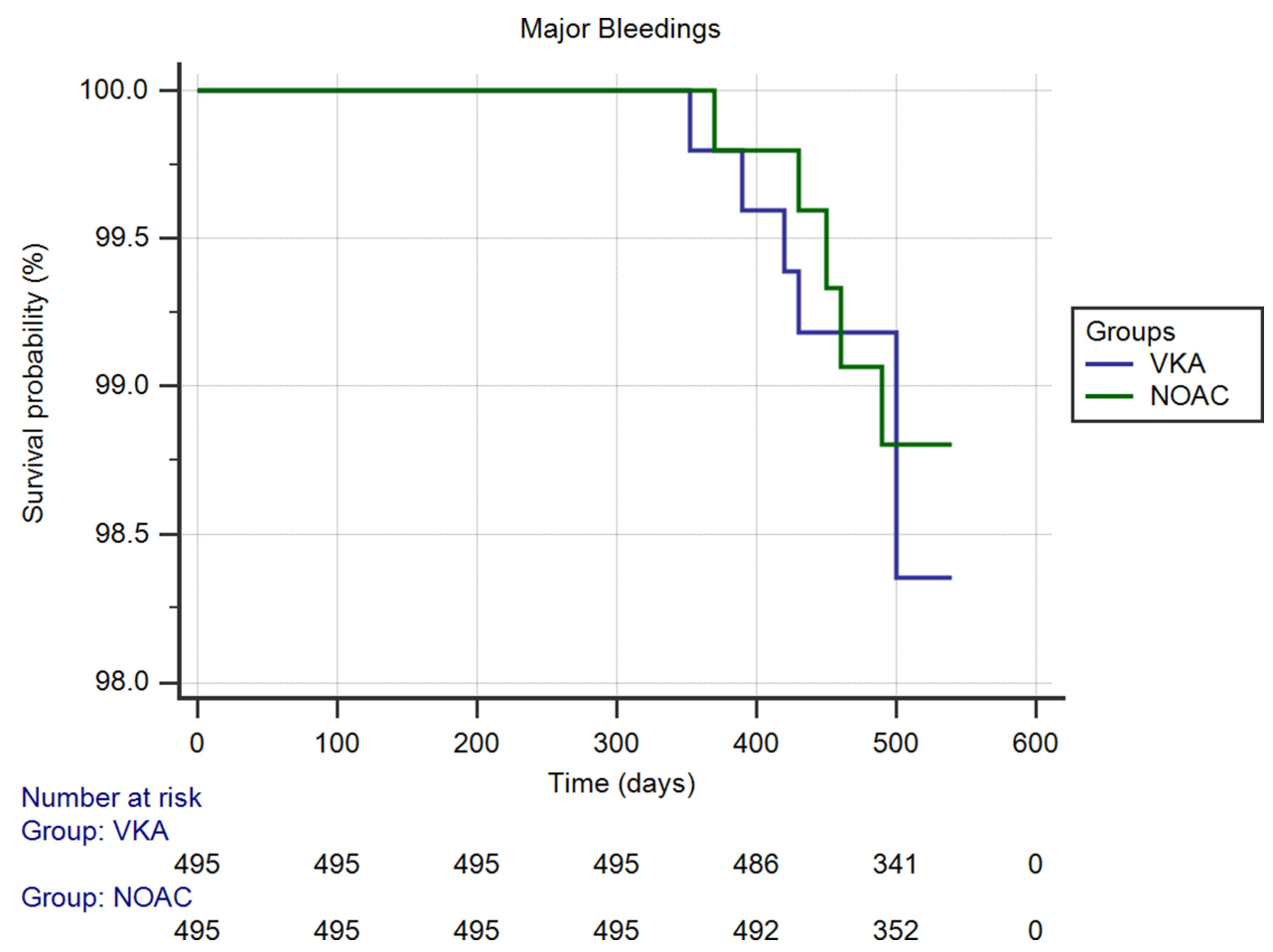

Figure I Kaplan Meier survival curve analysis estimating the risk of major bleedings in VKA and NOAC groups.

$\mathrm{DS}_{2} \mathrm{VASc}$ score: 6 and CC: 34 milliliter/minute in one 32milliliter/minute in one patient and INR 2.1; $\mathrm{CHA}_{2} \mathrm{DS}_{2}$ patient in DAB therapy]; and two patients $(0.4 \%)$ in the VASc score: 3; CC: 31 milliliter/minute in another VKAs group (INR: 2.2; $\mathrm{CHA}_{2} \mathrm{DS}_{2} \mathrm{VASc}$ c score: 4; CC: patient). The EC was performed within $23 \pm 2$ days. There

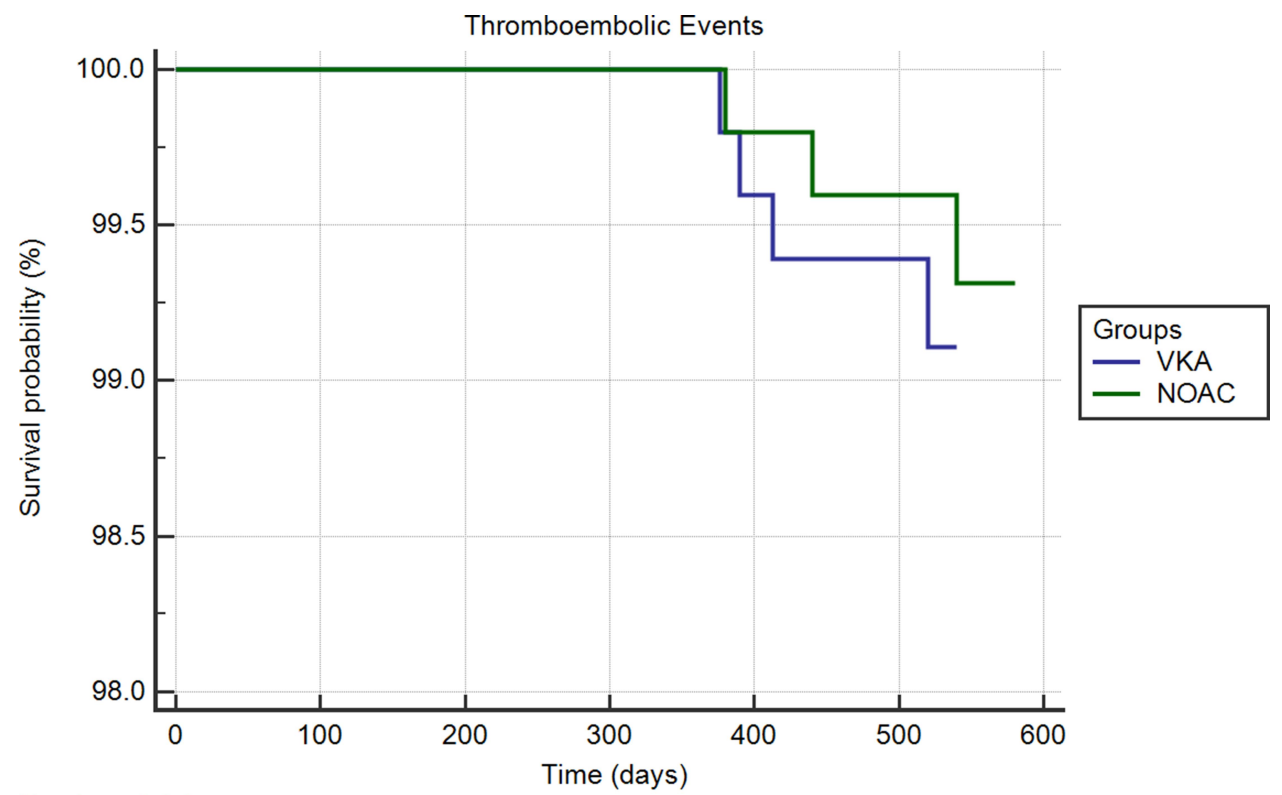

Number at risk Group: VKA

\begin{tabular}{|c|c|c|c|c|c|}
\hline Group: NOAC & 495 & 495 & 495 & 492 & 352 \\
\hline 495 & 495 & 495 & 495 & 494 & 356 \\
\hline
\end{tabular}

Figure 2 Kaplan Meier survival curve analysis estimating the risk of thromboembolic events in VKA and NOAC groups. 


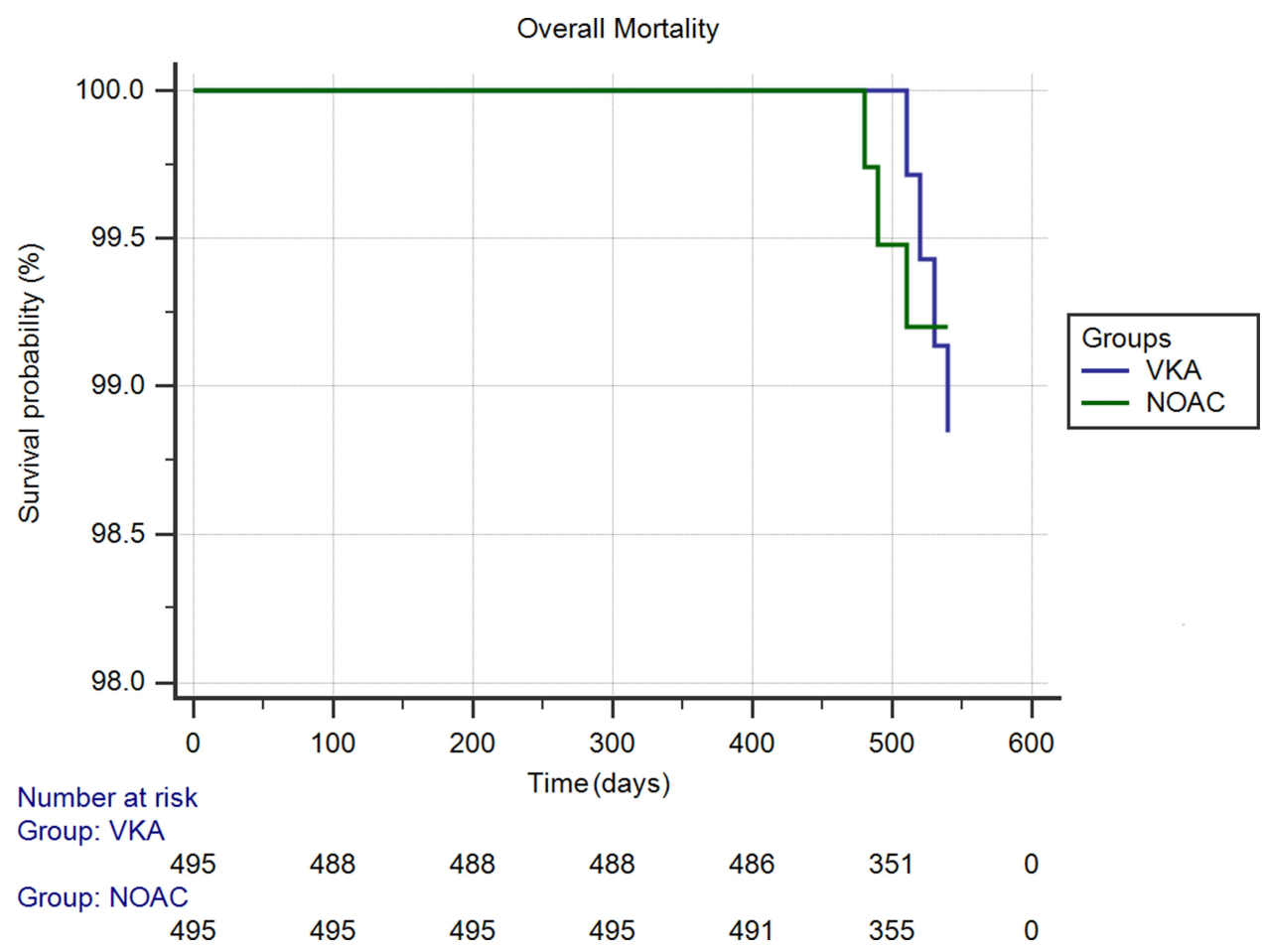

Figure 3 Kaplan Meier survival curve analysis estimating the risk of overall mortality in VKA and NOAC groups.

was an acute cardioversion success rate of $87.6 \%$ (n: $434 /$ 495 ) in the NOACs group and of $85.6 \%$ (n: 424/495) in the VKAs group $(P=0.35)$. All patients continued the anticoagulation treatment four weeks after EC, having a $\mathrm{CHA}_{2} \mathrm{DS}_{2}$ VASc score greater than 2 . The primary safety endpoint was experienced by 12 patients ( 5 in NOACs and 7 in VKAs). The cumulative MBs incidence was $1.01 \%$ in the NOACs group and $1.4 \%$ in the VKAs group $(P=0.5)$ (Figure 1). The intracranial hemorrhages (ICH) percentage was $0.2 \%$ in NOACs group $(1 / 495)$ and $0.4 \%$ in VKAs group $(P=0.6)$. The gastrointestinal bleedings (GIB) percentage was $0.8 \%$ in NOACs group $(4 / 495)$ and $1.01 \%$ in VKAs group $(P=0.7)$. The hemorrhagic event rates were $1.6 \%$ in Dabigatran group (n 2/119), $0.9 \%$ in rivaroxaban group (1/109), $0.8 \%$ in apixaban group (n $1 / 129), 0.7 \%$ in edoxaban group (n 1/138). The primary effectiveness endpoint was experienced by 7 patients ( 3 in NOACs and 4 in VKAs). The cumulative thromboembolic events incidence was $0.6 \%$ in NOACs group and $0.8 \%$ in VKAs group ( $P=$ 0.7 ) (Figure 2). The thromboembolic event rate was $0.8 \%$ in dabigatran group (n 1/119), $0.9 \%$ in rivaroxaban group (n 1/129), 0.7\% in apixaban group (n 1/129). There were not thromboembolic events in edoxaban group. We found no significant differences in terms of thromboembolic and hemorrhagic event rates between NOACs. The cumulative incidence of all-causes mortality was $0.6 \%$ (n: 3 ) in the
NOACs group and of $0.8 \%(n: 4)$ in VKAs group $(\mathrm{P}=0.7)$ (Figure 3). The cumulative incidence of CRNMB events was $1.2 \%(n: 6)$ in the NOACs group and $2.2 \%(n: 11)$ in the VKAs group $(P=0.2)$. The anticoagulant treatment discontinuation rate was $1.6 \%(\mathrm{~N}: 8)$ in the NOACs group and $3.6 \%(\mathrm{n}: 18)$ in the VKAs group $(P=0.04)$ (Table 2).

\section{Discussion}

The clinical performance of NOACs among AF patients scheduled for EC in real life setting represents an unmet medical need. Previous meta-analyses showed NOACs effectiveness and safety versus VKAs in the cardioversion setting including AF patients from randomized clinical trials (X-Vert, Ensure-AF and Emanate study). ${ }^{35-37}$ Our study assessed the NOACs safety and effectiveness versus VKAs treatment including only real-world patients with AF, scheduled for TEE-guided electrical cardioversion and followed for at least twelve months. Our experience did not show significant differences in the cumulative incidence of major bleedings, thromboembolic events, CRNMB and all-cause mortality between AF patients on NOACs compared to those on VKAs. Our real-world data confirmed the results of three previous RCTs (X-VeRT, ENSURE, EMANATE) which prospectively compared NOACs (rivaroxaban, edoxaban 
Table 2 Safety and Effectiveness Endpoints and Anticoagulant Treatment Discontinuation Rate in the NOACs and VKAs Groups

\begin{tabular}{|l|l|l|l|}
\hline \multirow{2}{*}{ Variable } & NOACs & VKAs & \multirow{2}{*}{$P$ value } \\
\cline { 2 - 3 } & $(n=495)$ & $(n=495)$ & \\
\hline Stroke/SE/TIA & $0.6 \%$ & $0.8 \%$ & 0.7 \\
Major Bleedings & $1.01 \%$ & $1.4 \%$ & 0.5 \\
All-cause mortality & $0.6 \%$ & $0.8 \%$ & 0.7 \\
CRNMB & $1.2 \%$ & $2.2 \%$ & 0.2 \\
ICH & $0.2 \%$ & $0.4 \%$ & 0.6 \\
GIB & $0.8 \%$ & $1.01 \%$ & 0.7 \\
Anticoagulant & $1.6 \%$ & $3.6 \%$ & 0.04 \\
therapy & & & \\
discontinuation rate & & & \\
\hline
\end{tabular}

Abbreviations: SE, systemic embolism; TIA, transient ischemic attack; CRNMB, clinically non relevant major bleedings; ICH, intracranial hemorrhages; GIB, gastrointestinal bleedings.

and apixaban) versus VKAs in patients undergoing AF cardioversion, ${ }^{24-26}$ supporting the evidence of their use in the real-world setting.

The optimal and persistent use of NOACs is of pivotal importance for the thromboembolic risk reduction in $\mathrm{AF}$ patients. Among our study population, the NOACs treatment was characterized by a lower discontinuation rate compared to VKAs therapy, confirming previous realworld studies data. ${ }^{38}$ Furthermore, low and similar incidences of left atrial appendage thrombus revealed by TEE were reported in NOACs $(0.4 \%)$ and VKAs $(0.4 \%)$ therapy groups, lower than those showed by previous prospective randomized studies in which the left atrial appendage thrombus percentage ranged from 3.5 to $8 \% .{ }^{25-27}$ These differences could be related to the different clinical features between our study cohort and those of randomized clinical trials. In particular, our patients showed a mean $\mathrm{CHA}_{2} \mathrm{DS}_{2} \mathrm{VASc}$ score $(2.3 \pm 0.4$ in NOACs group versus $2.2 \pm 0.5$ in VKAs group) lower than those of patients included in the RCTs[2.3 (1.6) in X-Vert study (24); 2-6 (SD 1.4) in Ensure-AF study; ${ }^{25} 2.4 \pm 1.7$ in Emanate study. ${ }^{26}$

The high thromboembolic risk and the moderate renal impairment might be clinical predictors of left atrial thrombus in real-world setting. Further studies on larger populations are necessary to confirm this hypothesis.

\section{Limitations}

The data collection performed in a single hospital represents a limitation of this study. The side effects detection is usually more accurate in RCTs than in real life monocenter observational studies. Since our study included mostly AF patients with low CHA2DS2VAsc score, conserved glomerular filtration rate and left ventricular ejection, our results cannot be generalized to the overall $\mathrm{AF}$ population undergoing electrical cardioversion. Moreover, the exclusion of VKA patients with low TTR could cause analysis bias in favour of VKA treatment. Therefore, further studies with larger sizes are needed to detect any eventual differences in haemorrhagic and thromboembolic events between patients on NOACs and those on VKAs treatment undergoing AF electrical cardioversion, given the low incidence of haemorrhagic and thromboembolic events with each of the two strategies.

\section{Conclusions}

The present propensity score-matched study showed a safe and effective clinical profile of NOACs in AF patients scheduled for electrical cardioversion with no statistically significant differences in the both bleeding and thromboembolic events incidences compared to VKAs. NOACs therapy was associated with lower discontinuation rate, supporting the hypothesis of a better compliance among AF patients undergoing EC.

\section{Disclosure}

The authors report no conflicts of interest in this work.

\section{References}

1. Airaksinen KE, Grönberg T, Nuotio I, et al. Thromboembolic complications after cardioversion of acute atrial fibrillation: the FinCV (Finnish CardioVersion) study. J Am Coll Cardiol. 2013;62 (13):1187-1192. doi:10.1016/j.jacc.2013.04.089

2. Connolly SJ, Ezekowitz MD, Yusuf S, et al. Dabigatran versus Warfarin in Patients with Atrial Fibrillation. N Eng J Med. 2009;361 (12):1139-1151. doi:10.1056/NEJMoa0905561

3. Giugliano RP, Ruff CT, Braunwald E, et al. Edoxaban versus warfarin in patients with atrial fibrillation. $N$ Engl $J$ Med. 2013;369 (22):2093-2104. doi:10.1056/NEJMoa1310907

4. Granger CB, Alexander JH, McMurray JJV, et al. Apixaban versus Warfarin in Patients with Atrial Fibrillation. $N$ Eng J Med. 2011;365 (11):981-992. doi:10.1056/NEJMoa1107039

5. Patel MR, Mahaffey KW, Garg J, et al. Rivaroxaban versus Warfarin in Nonvalvular Atrial Fibrillation. $N$ Eng $J$ Med. 2011;365 (10):883-891. doi:10.1056/NEJMoa1009638

6. Russo V, Bianchi V, Cavallaro C, et al. Efficacy and safety of dabigatran in a "real-life" population at high thromboembolic and hemorrhagic risk: data from MonaldiCare registry. Eur Rev Med Pharmacol Sci. 2015;19(20):3961-3967.

7. Russo V, Rago A, Proietti R, et al. Efficacy and safety of the target-specific oral anticoagulants for stroke prevention in atrial fibrillation: the real-life evidence. Ther Adv Drug Saf. 2017;8(2):67-75. doi: $10.1177 / 2042098616673990$ 
8. Russo V, Rago A, D'Onofrio A, et al. The clinical performance of dabigatran in the Italian real-life experience. J Cardiovasc Med. 2017;18(11):922-923. doi:10.2459/JCM.0000000000000548

9. Proietti R, Joza J, Arensi A, et al. Novel nonpharmacologic approaches for stroke prevention in atrial fibrillation: results from clinical trials. Med Devices. 2015;8:103-114. doi:10.2147/MDER. S70672

10. Melillo E, Carbone A, Rago A, et al. Update on Direct Oral Anticoagulants in Atrial Fibrillation Patients Undergoing Cardiac Interventional Procedures: from Clinical Trials to Real-World Evidence. $J$ Cardiovasc Pharmacol. 2020;75(3):185-199. doi:10.1097/FJC.0000000000000786

11. Russo V, Carbone A, Rago A, et al. Direct Oral Anticoagulants in Octogenarians With Atrial Fibrillation: it Is Never Too Late. J Cardiovasc Pharmacol. 2019;73(4):207-214. doi:10.1097/ FJC.0000000000000661

12. Russo V, Bottino R, Rago A, et al. Atrial Fibrillation and Malignancy: the Clinical Performance of Non-Vitamin K Oral Anticoagulants-A Systematic Review. Semin Thromb Hemost. 2019;45(2):205-214. doi:10.1055/s-0038-1661386

13. Russo V, Bottino R, Rago A, et al. Clinical Performance of Nonvitamin K Antagonist Oral Anticoagulants in Real-World Obese Patients with Atrial Fibrillation. Semin Thromb Hemost. 2020;46 (8):970-976. doi:10.1055/s-0040-1715792

14. Russo V, Paccone A, Rago A, et al. Apixaban in a Morbid Obese Patient with Atrial Fibrillation: a Clinical Experience Using the Plasmatic Drug Evaluation. J Blood Med. 2020;11:77-81. doi:10.2147/JBM.S229526

15. Russo V, Rago A, Papa A, et al. Use of Non-Vitamin K Antagonist Oral Anticoagulants in Atrial Fibrillation Patients with Malignancy: clinical Practice Experience in a Single Institution and Literature Review. Semin Thromb Hemost. 2018;44(4):370-376. doi:10.1055/ s-0037-1607436

16. Russo V, Attena E, Mazzone C, et al. Nonvitamin K Antagonist Oral Anticoagulants Use in Patients with Atrial Fibrillation and Bioprosthetic Heart Valves/Prior Surgical Valve Repair: a Multicenter Clinical Practice Experience. Semin Thromb Hemost. 2018;44(4):364-369. doi:10.1055/s-0037-1615261

17. Russo V, Carbone A, Attena E, et al. Clinical Benefit of Direct Oral Anticoagulants Versus Vitamin K Antagonists in Patients with Atrial Fibrillation and Bioprosthetic Heart Valves. Clin Ther. 2019;41 (12):2549-2557. doi:10.1016/j.clinthera.2019.10.008

18. Russo V, Attena E, Mazzone C, et al. Real-life Performance of Edoxaban in Elderly Patients With Atrial Fibrillation: a Multicenter Propensity Score-Matched Cohort Study. Clin Ther. 2019;41 (8):1598-1604. doi:10.1016/j.clinthera.2019.04.041

19. Russo V, Attena E, Rago A, et al. Clinical Outcome of Edoxaban vs. Vitamin K Antagonists in Patients with Atrial Fibrillation and Diabetes Mellitus: results from a Multicenter, Propensity-Matched, Real-World Cohort Study. J Clin Med. 2020;9(6):1621. doi:10.3390/ jcm9061621

20. Nagarakanti R, Ezekowitz MD, Oldgren J, et al. Dabigatran versus warfarin in patients with atrial fibrillation: an analysis of patients undergoing cardioversion. Circulation. 2011;123(2):131-136. doi:10.1161/CIRCULATIONAHA.110.977546

21. Piccini JP, Stevens SR, Lokhnygina Y, et al. Outcomes after cardioversion and atrial fibrillation ablation in patients treated with rivaroxaban and warfarin in the ROCKET AF trial. $J$ Am Coll Cardiol. 2013;61(19):1998-2006. doi:10.1016/j.jacc.2013.02.025

22. Flaker G, Lopes RD, Al-Khatib SM, et al. Efficacy and safety of apixaban in patients after cardioversion for atrial fibrillation: insights from the ARISTOTLE Trial (Apixaban for Reduction in Stroke and Other Thromboembolic Events in Atrial Fibrillation). J Am Coll Cardiol. 2014;63(11):1082-1087. doi:10.1016/j. jacc.2013.09.062
23. Plitt A, Ezekowitz MD, De Caterina R, et al. Cardioversion of Atrial Fibrillation in ENGAGE AF-TIMI 48. Clin Cardiol. 2016;39 (6):345-346. doi:10.1002/clc.22537

24. Cappato R, Ezekowitz MD, Klein AL, et al. Rivaroxaban vs. vitamin $\mathrm{K}$ antagonists for cardioversion in atrial fibrillation. Eur Heart J. 2014;35(47):3346-3355. doi:10.1093/eurheartj/ehu367

25. Goette A, Merino JL, Ezekowitz MD, et al. Edoxaban versus enoxaparin-warfarin in patients undergoing cardioversion of atrial fibrillation (ENSURE-AF): a randomised, open-label, phase $3 \mathrm{~b}$ trial. Lancet. 2016;388(10055):1995-2003. doi:10.1016/S0140-6736(16)31474-X

26. Ezekowitz MD, Pollack CV, Sanders P, et al. Apixaban compared with parenteral heparin and/or vitamin $\mathrm{K}$ antagonist in patients with nonvalvular atrial fibrillation undergoing cardioversion: rationale and design of the EMANATE trial. Am Heart J. 2016;179:59-68. doi:10.1016/j.ahj.2016.06.008

27. Steffel J, et al. The 2018 European Heart Rhythm Association Practical Guide on the use of non-vitamin K antagonist oral anticoagulants in patients with atrial fibrillation. Eur Heart J. 2018;39 (16):1330-1393

28. Hindricks G, et al. ESC Guidelines for the diagnosis and management of atrial fibrillation developed in collaboration with the European Association for Cardio-Thoracic Surgery (EACTS). Eur Heart J. 2020;42(5):373-498.

29. Russo V, Rago A, Papa AA, et al. Efficacy and safety of dabigatran in patients with atrial fibrillation scheduled for transoesophageal echocardiogram-guided direct electrical current cardioversion: a prospective propensity score-matched cohort study. J Thromb Thrombolysis. 2018;45(2):206-212. doi:10.1007/s11239-017-1599-5

30. Rago A, Papa AA, Cassese A, et al. Clinical Performance of Apixaban vs. Vitamin $\mathrm{K}$ Antagonists in Patients with Atrial Fibrillation Undergoing Direct Electrical Current Cardioversion: a Prospective Propensity Score-Matched Cohort Study. Am J Cardiovasc Drugs. 2019;19(4):421-427. doi:10.1007/s40256-019-00341-9

31. Rago A, Papa AA, Attena E, et al. Direct Current Cardioversion in Atrial Fibrillation Patients on Edoxaban Therapy Versus Vitamin K Antagonists: a Real-world Propensity Score-Matched Study. Cardiovasc Drugs Ther. 2020. doi:10.1007/s10557-020-07078-7

32. Stabile G, Russo V, Rapacciuolo A, et al. Transesophageal echocardiograpy in patients with persistent atrial fibrillation undergoing electrical cardioversion on new oral anticoagulants: a multi center registry. Int J Cardiol. 2015;184:283-284. doi:10.1016/j.ijcard.2015.02.075

33. Bertaglia E, Anselmino M, Zorzi A, et al. NOACs and atrial fibrillation: incidence and predictors of left atrial thrombus in the real world. Int J Cardiol. 2017;249:179-183. doi:10.1016/j.ijcard.2017.07.048

34. Kaatz S, Ahmad D, Spyropoulos AC, et al. Definition of clinically relevant non-major bleeding in studies of anticoagulants in atrial fibrillation and venous thromboembolic disease in non-surgical patients: communication from the SSC of the ISTH. $J$ Thromb Haemost. 2015;13(11):2119-2126. doi:10.1111/jth.13140

35. Telles-Garcia N, Dahal K, Kocherla C, et al. Non-vitamin $\mathrm{K}$ antagonists oral anticoagulants are as safe and effective as warfarin for cardioversion of atrial fibrillation: a systematic review and meta-analysis. Int $J$ Cardiol. 2018;268:143-148. doi:10.1016/j. ijcard.2018.04.034

36. Brunetti ND, Tarantino N, De Gennaro L, et al. Direct oral anti-coagulants compared to vitamin-K antagonists in cardioversion of atrial fibrillation: an updated meta-analysis. $J$ Thromb Thrombolysis. 2018;45(4):550-556. doi:10.1007/s11239-018-1622-5

37. Kotecha D, Pollack CV, De Caterina R, et al. Direct Oral Anticoagulants Halve Thromboembolic Events After Cardioversion of AF Compared With Warfarin. J Am Coll Cardiol. 2018;72 (16):1984-1986. doi:10.1016/j.jacc.2018.07.083

38. Verdecchia P, D’Onofrio A, Russo V, et al. Persistence on apixaban in atrial fibrillation patients: a retrospective multicentre study. J Cardiovasc Med. 2019;20(2):66-73. doi:10.2459/ JCM.0000000000000744 


\section{Publish your work in this journal}

The Journal of Blood Medicine is an international, peer-reviewed, open access, online journal publishing laboratory, experimental and clinical aspects of all aspect pertaining to blood based medicine including but not limited to: Transfusion Medicine; Blood collection, Donor issues, Transmittable diseases, and Blood banking logistics; Immunohematology; Artificial and alternative blood based therapeutics; Hematology; Biotechnology/nanotechnology of blood related medicine; Legal aspects of blood medicine; Historical perspectives. The manuscript management system is completely online and includes a very quick and fair peer-review system. Visit http://www.dovepress.com/testimonials.php to read real quotes from published authors. 